\title{
ASSESSMENT OF CONCRETE MATURITY LEVEL USING P-WAVE RESONANCE USING FINITE ELEMENT ANALYSIS
}

\author{
K. Sivasubramanian ${ }^{1}$, R. Ravichandran ${ }^{2}$, K.N. Lakshmikandhan ${ }^{3}$, P. Sivakumar ${ }^{4}$ \\ ${ }^{I}$ Scientist, ACTEL, CSIR-Structural Engineering Research Centre, Chennai, India \\ ${ }^{2}$ Scientist, ACTEL, CSIR-Structural Engineering Research Centre, Chennai, India \\ ${ }^{3}$ Scientist, ACTEL, CSIR-Structural Engineering Research Centre, Chennai, India \\ ${ }^{4}$ Scientist, ACTEL, CSIR-Structural Engineering Research Centre, Chennai, India
}

\begin{abstract}
With the recent developments in the non-destructive testing methods, the possibility to assess the changes in material characteristics caused by the addition of admixtures, influence of temperature, and other parameters are gaining significance. Towards this, the present work focuses on using the resonance test method for the experimental investigation. In the case of transient dynamic analysis which involves vibrations at higher frequencies, the understanding obtained from finite element simulations is relatively higher to that gained by conducting experiments. Hence, the present work mainly aims to analytically model the concrete specimens as it matures during the first 28 days after concreting. Towards this, the modelling of the specimens and the adaptation of the appropriate boundary conditions to simulate the resonance test method becomes significant. The results of the experimental and finite element analysis are compared and found to be in good agreement.
\end{abstract}

Keywords: Finite element analysis, concrete, maturity level, $P$-wave resonance, vibration characteristics

\section{INTRODUCTION}

Recently, research on test procedures that are sensitive to minor changes in the material properties are gaining greater significance. Such test procedures would be very useful to investigate the concrete maturity level, monitor the concrete setting process, influence of admixtures, freezing-thawing effect, degradation to fire, and many others. Towards this, the American Standards for Testing Methods has recommended a test procedure based on directional modal frequency ${ }^{1}$. Here, the elastic stress wave travels in the test medium causing compression and tension wave components along the direction of wave propagation resulting in a lamb wave resonance.

ASTM C $215^{1}$ recommends the resonance test as a technique to assess the variation in the dynamic elastic modulus. The resonance test method uses directional modal characteristics to evaluate the material characteristics by assessing the dynamic elastic modulus. The test method has been popularly used to assess the dynamic elastic modulus ${ }^{2}$, influence of admixtures ${ }^{3}$, influence of loading ${ }^{4}$, deterioration due to fatigue ${ }^{5}$, freezing-thawing effect ${ }^{6}$, effect of deicers on concrete deterioration ${ }^{7}$, monitor self-healing process in concrete $^{8}$, influence of high temperature ${ }^{9}$, and many more. The earlier research works have demonstrated that the resonance test method is a very useful technique investigating deterioration or improvement in concrete characteristics $^{10,11}$.

Finite element simulations have always played a significant role alongside the experimental investigations to understand and validate the results with respect to the theoretical concepts. Finite element simulations are very useful in improving the understanding of vibration problems at higher frequencies. Recently, the finite element modeling of the resonance test methods has been of interest to the researchers. Haecker et $\mathrm{al}^{12}$ have employed finite element modeling of the microstructure of concrete to simulate the resonance test method. $\mathrm{Lu}$ et $\mathrm{al}^{13}$ employed finite element analysis to validate the experimental program on the effect of different types of aggregates in concrete. The present work primarily aims at numerically simulating the resonance test method for assessing the directional resonance characteristics.

In the present work, it is aimed to assess the maturity of concrete by simulating the lamb wave resonance in concrete specimens. Towards this, two concrete mixes have been considered. The first mix is a normal strength concrete and the second is a high strength concrete. The results from the experimental study have been compared validated with the finite element simulation results. The results indicate that the finite element analysis can be effectively used to simulate lamb wave resonance in concrete specimens and thus aid in assessing the maturity of the concrete for the twenty-eight days after concreting.

\section{RESONANCE TEST METHOD}

Resonance test is a non-destructive evaluation method gaining popularity for assessing the material characteristics, especially those that changes with time. The procedure for this test method is standardised and recommended in the guidelines of ASTM C215 ${ }^{1}$. The resonance test relies on the evaluation of the fundamental frequency along a particular direction to evaluate the properties of the test medium in that direction. The ASTM standard ${ }^{1}$ recommends the use of 
longitudinal, transverse and torsional modal frequencies in the fundamental mode to evaluate the dynamic modulli of the specimen along those directions, respectively. The fundamental frequency used in this method is different from the more popular parameter, namely the whole body modal frequency.

The resonance test is being carried out in two ways. The distinction is mainly based on the type of source used to excite the medium. In the first method, an automatic pulser is used to impart the source waves into the test medium. This is called the forced resonance test. The second method is much simpler as it uses a hammer to produce the source waves. This method is called the impact resonance test and is being preferred over the first method for its simplicity. In the present work, the impact resonance test has been used. A schematic of the two types of resonance tests are shown in Fig. 1 and Fig. 2. Even though the method used for the generation of source waves are different, both the methods produce the same resonant frequency. This is because the focus is on assessing the frequency of resonance which depends on the frequency content of the source wave and not on it's the amplitude.

The resonance test method is being preferably used in the lower half of the audible frequency band, that is, between the 100 to $10,000 \mathrm{~Hz}$. The accelerometer used in this test is sensitive to capture frequencies upto $20 \mathrm{kHz}$. Hence, tests can be carried out covering the full range of the audible frequency. In this test, the oscilloscope captures the vibrations of the test specimen. The frequency analyser is used to convert the time domain signal to the frequency spectrum where the dominant frequency corresponds to the fundamental mode of the test medium along the direction of investigation. Fig. 3 and Fig. 4, respectively, show the instrumentation and the arrangement for evaluating the longitudinal mode using the resonance test method.

In the present work, it is preferred to use the longitudinal resonance frequency alone for the evaluation of the dynamic elastic modulus of the concrete medium. The dynamic elastic modulus of the test medium can be evaluated using the equation ${ }^{1}$

$$
E_{d y n}=D M\left(n^{\prime}\right)^{2}
$$

In the resonance test method, one-half of the wavelength participates in resonating the test specimen in the longitudinal fundamental mode. This fundamental modal frequency is related to the velocity of the wave in the test medium by the equation

$$
f=v / 2 d_{1}
$$

Here, the velocity of the wave in the test medium and can be evaluated using the equation

$$
v=\sqrt{\frac{E_{d y n}(1-v)}{\rho(1+v)(1-2 v)}}
$$

Thus, using the longitudinal resonant frequency the variation in the dynamic elastic modulus can be assessed using the resonance test method.

\section{RESULTS AND DISCUSSIONS}

The present work primarily aims at analytically simulating the resonance test method. Towards this, a prismatic beam has been considered. It is first proposed to demonstrate the difference in the whole body and directional modal characteristics using this problem. The analysis module used for both the analysis is the free vibration analysis. Also, the mesh size and the material properties have been kept constant in these problems. But, appropriate boundary conditions have been applied to bring out the particular type of modal characteristics from the beam. The results of the finite element analysis have been compared with the results from the classical equations to validate the finite element model. Later, the cube specimen which was considered for experimental evaluation of the concrete maturity level has been considered. The finite element model has been used to model the maturity level of concrete. The finite element results have been validated with the experimental results.

\subsection{Finite Element Modeling and its Validation}

Firstly, a prismatic beam of size $1.0 \times 0.1 \times 0.1 \mathrm{~m}$ is considered to validate the finite element model and to highlight the difference between the whole body modal vibration and the longitudinal modal resonance characteristics. It is assumed that the beam is made of concrete with characteristic compressive strength of 25 $\mathrm{MPa}$. The density of the concrete is taken as $2400 \mathrm{~kg} / \mathrm{m}^{3}$ and the elastic modulus is taken as $25000 \mathrm{~N} / \mathrm{mm}^{2}$. The finite element model has been created using a standard commercial finite element package. The mesh size of $10 \times 10 \times 10 \mathrm{~mm}$ has been chosen for the investigation. To evaluate the whole body modal characteristics, one end of the beam is fixed by constraining all the six degree of freedom on that face of the beam. The finite element model of the cantilever beam, the boundary conditions and meshing details used for the analysis are shown in Fig. 5. Free vibration analysis is performed to evaluate the modal characteristics. The whole body modal characteristics are obtained and the first three modal frequencies are found to be 52.16, 326.89 and $915.39 \mathrm{~Hz}$, respectively. The modal shapes corresponding to the first three modes are shown in Fig. 6. The modal frequencies obtained using finite element analysis is compared with the Timoshenko equation in Table 1. It has been observed that the errors are very small, thus validating the finite element model.

Now, the same prismatic beam is analysed for the longitudinal modal characteristics. It is proposed to simulate the test conditions in the resonance test. For this analysis, the mesh size and the material properties are maintained a constant. To evaluate the longitudinal modal frequencies the free vibration analysis module in the finite element package has been used. But the use of conventional boundary conditions would allow the specimen model to vibrate in its flexural and torsional modes. The need here is to evaluate 
the longitudinal modal characteristics. Towards this trials have been carried out before arriving at the appropriate boundary conditions for obtaining the longitudinal modal characteristics.

In this case, the beam is constrained such that the longitudinal mode is excited as the fundamental mode. For this analysis, all degrees of freedom on the left face of the beam are constrained except the translation degree of freedom along the longitudinal axis of the beam. The four sides along the longitudinal axis of the beam are constrained for translation along the axis orthogonal to the face of the beam. The boundary conditions applied on the beam is shown in Fig. 7. Now, the beam is analysed for longitudinal modes using free vibration analysis module. The first three longitudinal modal frequencies have been found to be 1701.0, 3401.5, 5101.2 Hz. The first three longitudinal mode shapes of the beam are shown in Fig. 8. From this figure, it can be observed that the longitudinal modes correspond to vibration characteristics of the beam dominated by compression and elongation of the beam along its longitudinal direction. Here too, the modes differ in the number of nodes or points of zero displacement that occur in the mode shape.

From Fig. 8, it can be observed that the first longitudinal mode represents the modal resonance that occurs during the resonance test. Henceforth, the fundamental longitudinal mode shall be considered for further investigation. The longitudinal modal frequencies of the beam are compared with those from the classical Equations (2) and (3) and the values are listed in Table 2 . It can be observed that the longitudinal modal frequency evaluated using the finite element model is in good agreement with the classical equations with errors less than $1 \%$. Thus, the finite element model is validated.

\subsection{Assessment of Concrete Maturity Level using}

\section{Resonance Test Method}

An experimental program has been carried out using resonance test on two concrete mixes. The preliminary tests on the cement, sand and coarse aggregates have been conducted as per Indian Standard codal recommendations. The fine aggregate used in the present work has been observed to fall in Grade III as per IS- $383^{14}$. The coarse aggregates confirm to the IS- $383^{14}$. Potable water has been used for making the concrete. OPC cement of 53 grade confirming to IS- $12269^{15}$ has been used. In the concrete mixes, the first mix is a Normal Strength Concrete and the next is a High Strength Concrete. The concrete mix has been designed as per the IS-10262 ${ }^{16}$ and ACI $211.4 \mathrm{R}^{17}$. A few trial mixes have been made before arriving at the final mix. The concrete mix ratio for the normal strength concrete is $1: 2.2: 3.1$ with water-cement ratio of 0.55 . The concrete mix ratio for the high strength concrete is $1: 1.4: 2.6$ with $\mathrm{w} / \mathrm{c}$ ratio of 0.35 along with the CONPLAST superplasticizer of $0.7 \%$ by weight of the cement.
For conducting the experiments, nine cube specimens of size $150 \times 150 \times 150 \mathrm{~mm}$ have been cast. On each day resonance test has been conducted on all the specimens. The average of the resonance frequencies obtained from all the specimens has been computed. With this the development of the concrete maturity level has been assessed.

\subsection{Finite Element Modeling of the Maturity Level} of Concrete

Now, the finite element analysis is carried out to evaluate the resonant longitudinal frequency for different levels of concrete maturity assessed using the experimental study. For this, the dynamic elastic modulus of the concrete on each day is evaluated using the longitudinal resonance frequency for both the concrete mixes. This value of the dynamic elastic modulus has been used in the material model in the finite element analysis. From the experiment, the density of NSC and HSC specimens has been found to be $2400 \mathrm{~kg} / \mathrm{m}^{3}$ and $2450 \mathrm{~kg} / \mathrm{m}^{3}$, respectively, and the same has been used in the finite element analysis. Poisson's ratio has been taken as 0.2 . The longitudinal resonance frequencies obtained from the experiment are compared with those obtained from finite element analysis in Fig. 9 and Fig. 10 for the NSC and HSC, respectively. It can be observed that the longitudinal resonance frequency obtained from the experimental and finite element studies are in good agreement. Thus, the theoretical finite element analysis validates the experimental investigation.

\section{CONCLUSION}

Evaluation of material properties and their variation is becoming more important these days. The resonance test is a simple test becoming popular for such investigations. The sensitivity of the method to identify variation in the material property using longitudinal resonance is well established in the literature. The present work focuses on conducting the finite element simulation of the longitudinal resonance of the test mediums and hence validates the experimental results.

Towards this, the work primarily focused on creating finite element models and to identify the appropriate boundary conditions to simulate the longitudinal resonance. In order to make the finite element model vibrate primarily along the longitudinal axis, the sides of the beam were constrained against translation in the transverse direction. The longitudinal modal characteristics have been compared with the theoretical values to validate the finite element model.

Then, experimental studies have been carried out using two concrete mixes, namely NSC and HSC. Cubes have been cast using these two mixes. Resonance test was carried out on the cube specimens. Later, the finite element model of the cube is developed and the material properties obtained from the experimental test have been used to evaluate the resonance frequencies for all the 28 days for the two types of concrete. The results of the FEA have been found to compare well with the experimental analysis. 


\section{ACKNOWLEDGEMENTS}

The paper is published with the permission of the Director, CSIR-SERC.

\section{NOTATIONS}

$v \quad$ - $\quad$ velocity of the wave

$\rho \quad$ - $\quad$ density of the test medium

$d_{1} \quad$ - depth of the test medium along the direction of wave propagation

$D \quad-\quad=5.093\left(L / d^{2}\right), \mathrm{m}^{-1}$ for a cylinder, or $=4$ $(L / b t), \mathrm{m}^{-1}$ for a prism.

D - $\quad$ diameter of cylinder, $\mathrm{m}$,

$E_{d y n} \quad$ - $\quad$ dynamic elastic modulus

$f \quad$ - $\quad$ fundamental modal frequency

$f_{w b} \quad-\quad$ whole body modal characteristics

HSC - High Strength Concrete

IS - - Indian Standard

L $\quad$ - $\quad$ length of specimen, m,

$\mathrm{M} \quad$ - $\quad$ mass of the specimen, $\mathrm{kg}$,

$n^{\prime} \quad$ - $\quad$ fundamental longitudinal frequency, $\mathrm{Hz}$,

NSC - $\quad$ Normal Strength Concrete

$\mathrm{t}, \mathrm{b} \quad$ - dimensions of cross section of prism, $\mathrm{m}, \mathrm{t}$ being the dimension in the direction in which it is driven),

$v \quad$ - $\quad$ Poisson's ratio of the test medium

w/c - water-cement ratio

\section{REFERENCES}

[1] ASTM C215, "Standard Test Method for Fundamental Transverse, Longitudinal, and Torsional Resonant Frequencies of Concrete Specimens", ASTM International, USA, 2008.

[2] Plachy, T, Padevet, P, Polak, M, "Comparison of Two Experimental Techniques for Determination of Young's Modulus of Concrete Specimens", Proc. Recent Advances In Applied And Theoretical Mechanics, Montreux, Spain, Dec 14-16, 2009, pp. 68-71.

[3] V. Giner, S. Ivorra, F.J. Baeza, B. Ferrer, "Effect of different admixtures on dynamic structural behavior of fiber reinforced concrete elements", Proc. of the 8th International Conference on Structural Dynamics, EURODYN 2011, Leuven, Belgium, 4-6 July 2011, G. De Roeck, G. Degrande, G. Lombaert, G. Muller (eds.), pp. 3245-3251

[4] Malaikah, A, Al-Saif, K and Al-Zaid, R, "Prediction of the dynamic modulus of elasticity of concrete under different loading conditions", Proc. of International Conference On Concrete Engineering and Technology, Universiti Malaya, 2004, pp. 7.

[5] Gheorghiu, C, Rhazi, JE, Labossière, P, "Impact resonance method for fatigue damage detection in reinforced concrete beams with carbon fibre reinforced polymer", Canadian Journal of Civil Engineering, 02/2011; 32(6), pp. 1093-1102. DOI: 10.1139/105-064

[6] Tikalsky, PJ, Pospisil, J, McDonald, W, "A method for assessment of the freeze-thaw resistance of preformed foam cellular concrete", Cement and Concrete Research, V. 34, No. 5, 2004, pp. 889-893.
[7] Darwin, D, Browning, JA, Gong, L, and Hughes, SR, "Effects of Deicers on Concrete Deterioration", Materials Journal, V. 105, No. 6, 2008, pp. 622-627.

[8] Li, VC, Yang, EH, "Self Healing in Concrete Materials, Self Healing Materials", Springer Series in Materials Science, V. 100, 2007, pp. 161-193.

[9] L. T. Phan, J. R. Lawson, F. L. Davis, "Effects of elevated temperature exposure on heating characteristics, spalling, and residual properties of high performance concrete", Materials and Structures, V. 34, No. 2, 2001, pp. 83-91.

[10] Lamond, JF, Pielert, JH, "Significance of Tests and Properties of Concrete and Concrete-making Materials", Issue 169, Part 4, ASTM International, USA, 2006, pp. 664.

[11] Ramachandran, VS, Beaudoin, JJ, "Handbook of Analytical Techniques in Concrete Science and Technology: Principles, Techniques and Applications”, Elsevier, 2000, pp. 1003.

[12] Haecker, C.-J, Garboczi, E.J, Bullard, J.W, Bohn, R.B, Sun, Z, Shah, SP, Voigt, T, "Modeling the linear elastic properties of Portland cement paste", Cement and Concrete Research, V. 35, No. 10, 2005, pp. 1948-1960.

[13] Lu, X, Sun, Q, Feng, W, Tian, J, "Evaluation of dynamic modulus of elasticity of concrete using impact-echo method", Construction and Building Materials, V. 47, 2013, pp. 231-239.

[14] IS 383, "Indian Standard for Specification for coarse and fine aggregates from natural sources for concrete", Bureau of Indian Standards, New Delhi, 1970 (Reaffirmed 2007).

[15] IS 12269, "Indian Standard for Specification for 53 grade ordinary Portland cement" Bureau of Indian Standards, New Delhi, 1987 (Reaffirmed 2008).

[16] IS 10262, "Indian Standard Recommended guidelines for concrete mix design”, Bureau of Indian Standards, New Delhi, 1982 (Reaffirmed 2005).

[17] ACI 211.4R-08, “Guide for Selecting Proportions for High-Strength Concrete with Portland Cement and Fly Ash”, ACI Committee 211, American Concrete Institute, USA, 2008. 


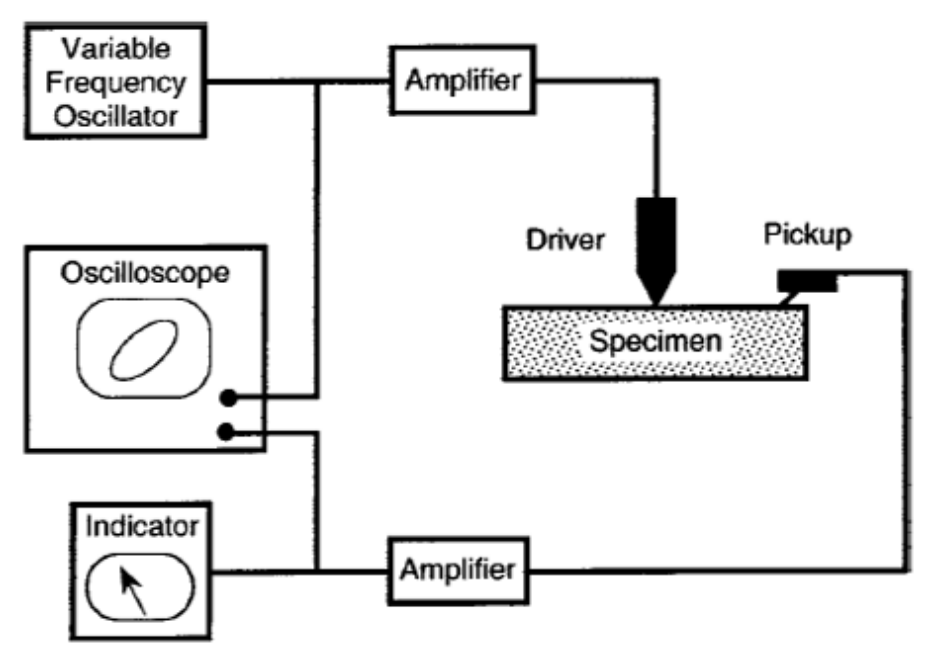

Fig. 1. Schematic of Apparatus for Forced Resonance Test

\section{Waveform Analyzer \\ or \\ Frequency Counter}
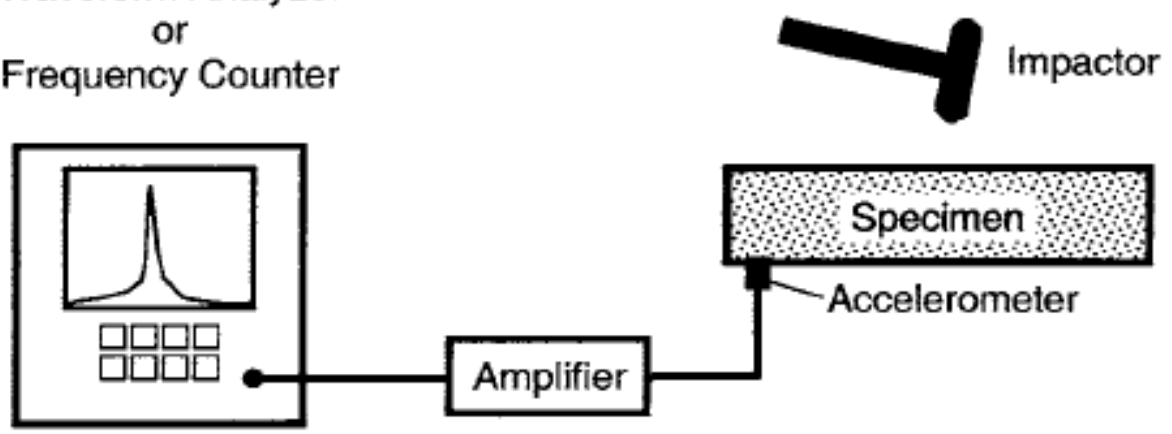

Fig. 2. Schematic of Apparatus for Impact Resonance Test

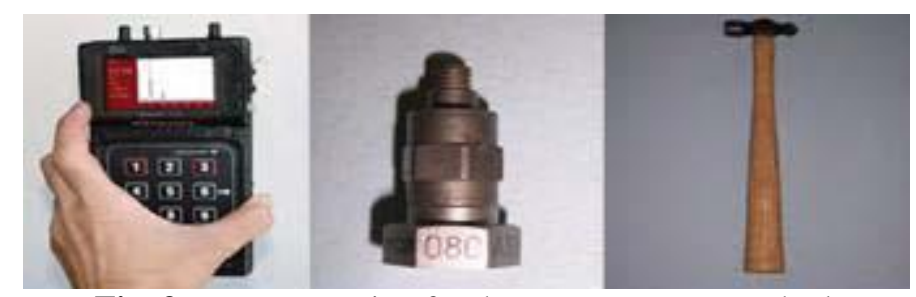

Fig. 3. Instrumentation for the resonance test method

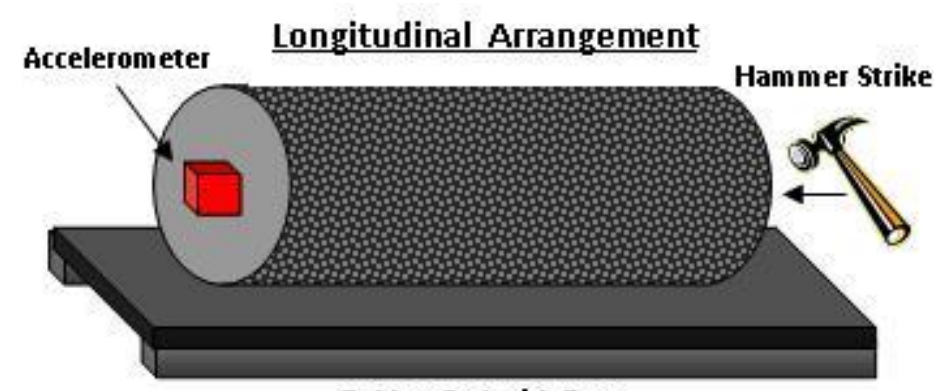

Rubber Pad with Feet

Fig. 4. Experimental arrangement for capturing longitudinal resonant frequency 


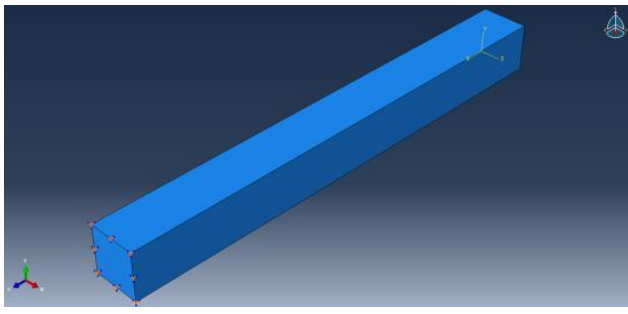

(a) FE model

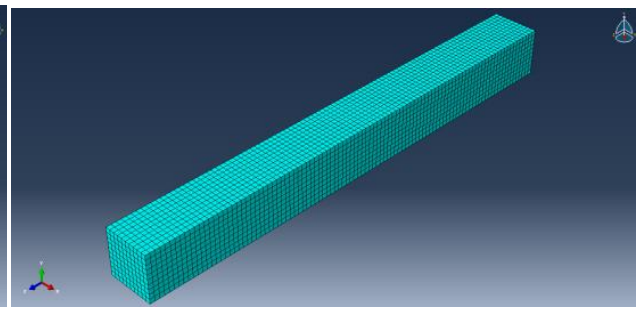

(b) Mesh

Fig. 5. Finite element model and meshing of the cantilever beam

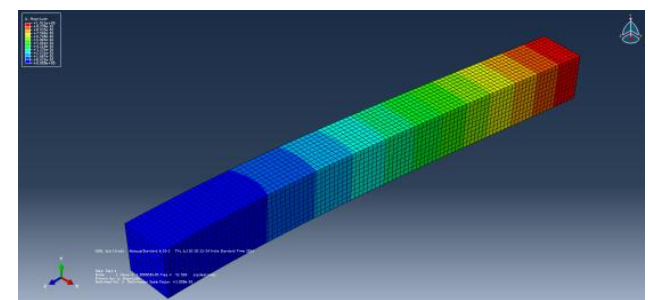

(a) Mode 1

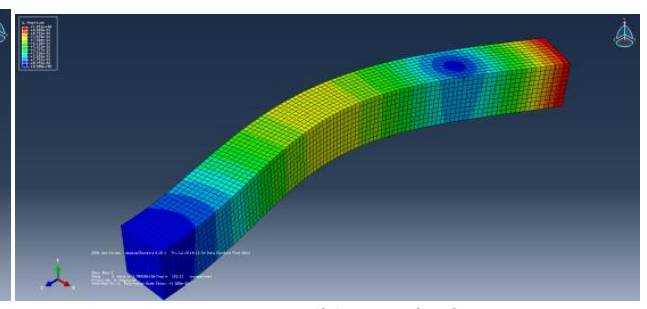

(b) Mode 2

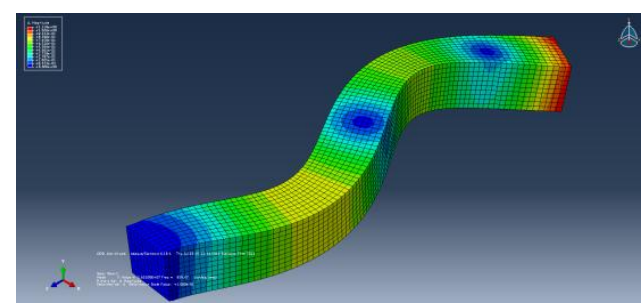

(c) Mode 3

Fig. 6. First three modes of the cantilever beam

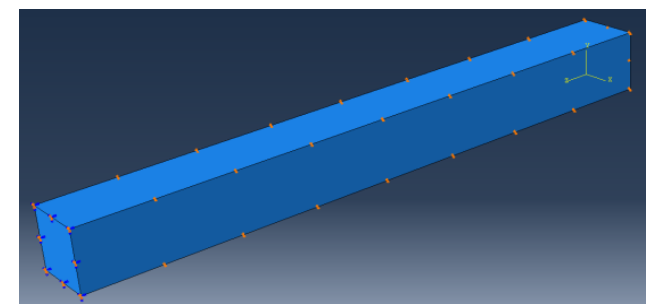

Fig. 7. Boundary conditions used to evaluate the longitudinal modal characteristics

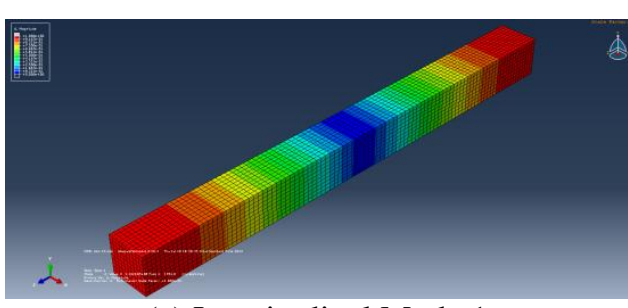

(a) Longitudinal Mode 1

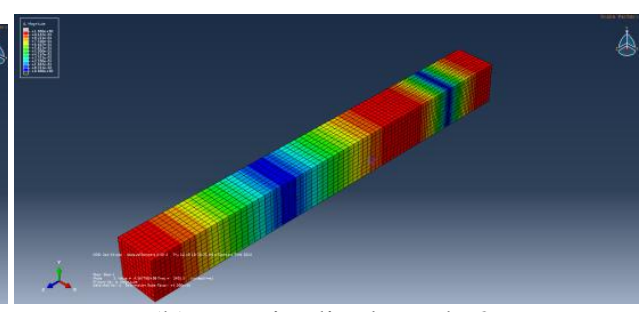

(b) Longitudinal Mode 2

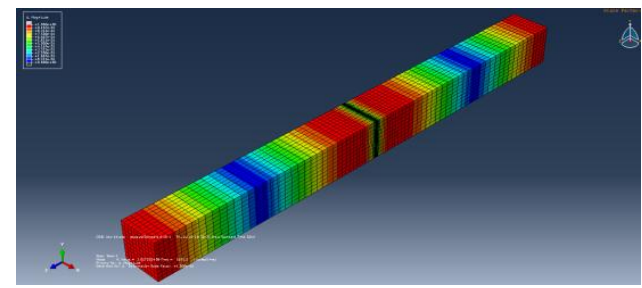

(c) Longitudinal Mode 3

Fig. 8. First three longitudinal modes of the prismatic beam 


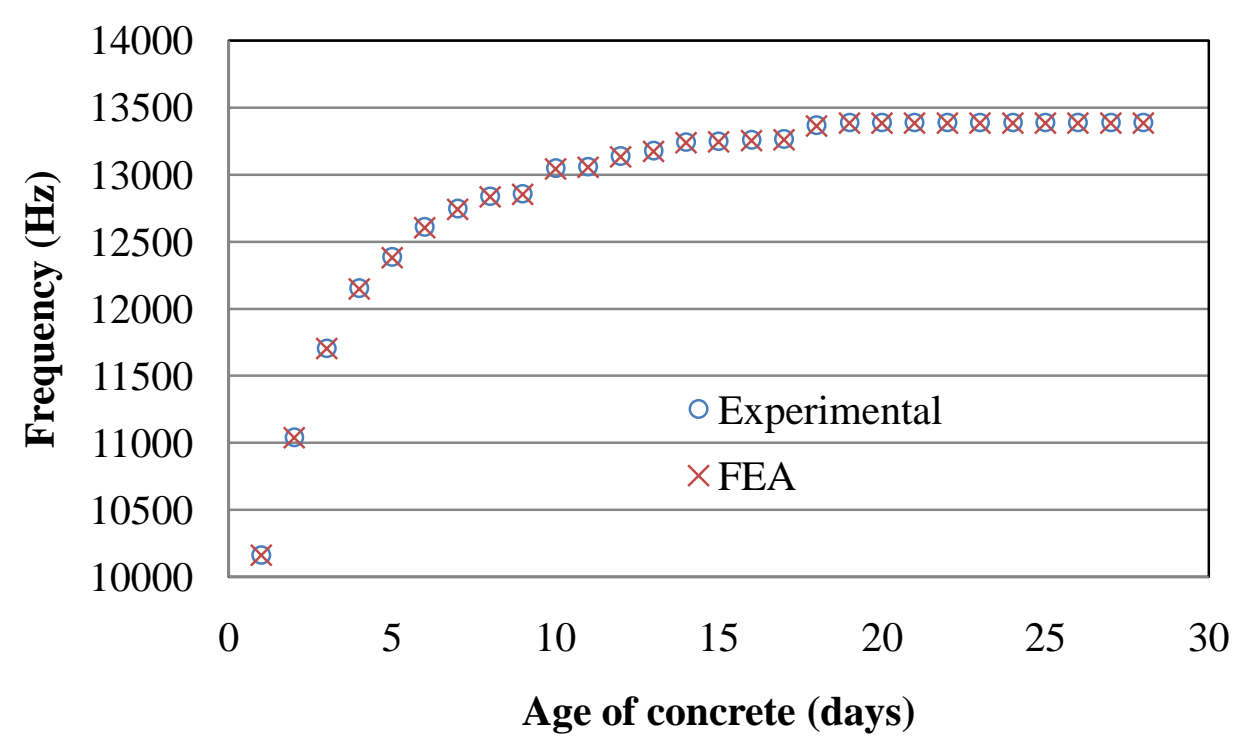

Fig. 9. Validation of the finite element model with the experimental data for NSC

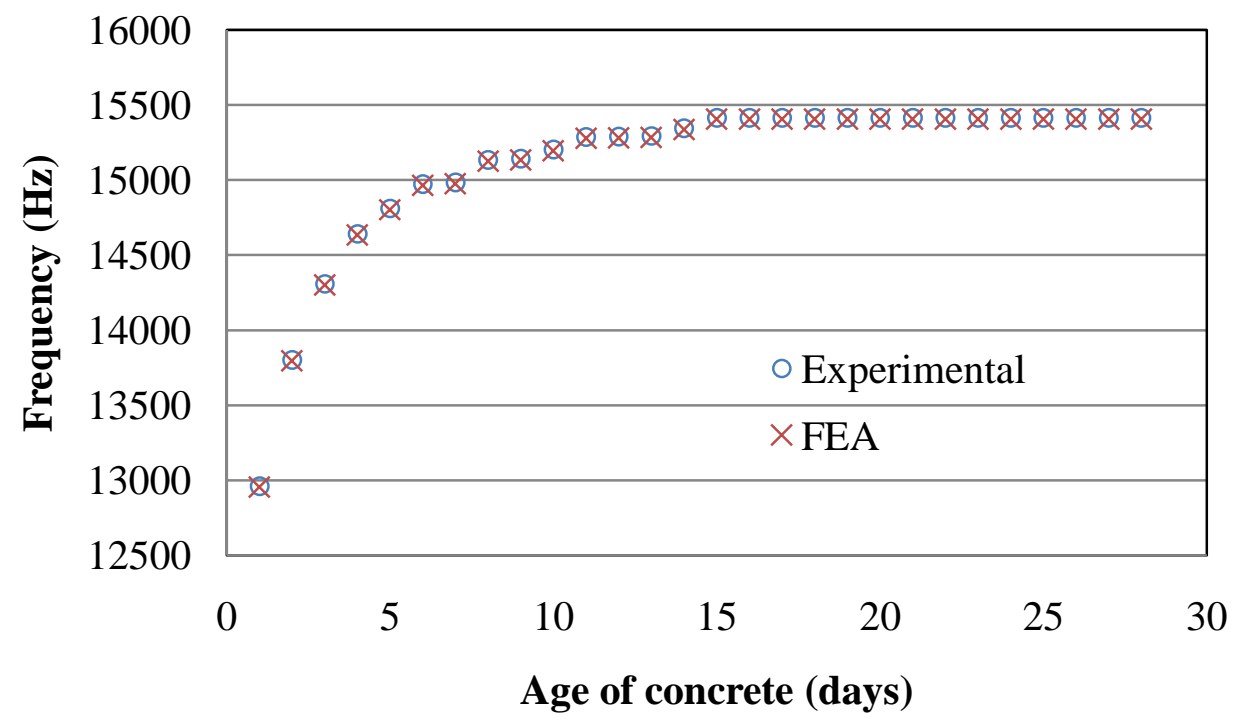

Fig. 10. Validation of the finite element model with the experimental data for HSC

Table 1. Comparison of the first three modal frequencies from FEA with classical Timoshenko beam characteristics

\begin{tabular}{|l|l|l|l|}
\hline Mode number & $\begin{array}{l}\text { Finite Element } \\
\text { Analysis }(\mathrm{Hz})\end{array}$ & $\begin{array}{l}\text { Timoshenko } \\
\text { equation }(\mathrm{Hz})\end{array}$ & Error $(\%)$ \\
\hline 1 & 52.16 & 51.595 & 1.09 \\
\hline 2 & 326.89 & 310.23 & 5.37 \\
\hline 3 & 915.39 & 819.47 & 11.71 \\
\hline
\end{tabular}

Table 2. Comparison of the two types of modal characteristics of the cantilever beam with theoretical values

\begin{tabular}{|l|l|l|l|}
\hline Mode number & \multicolumn{2}{|l|}{ Longitudinal frequency $(\mathrm{Hz})$} & Error $(\%)$ \\
\hline & FEA & Theoretical value & \\
\hline 1 & 1701.0 & 1701.035 & 0.002 \\
\hline 2 & 3401.5 & 3402.07 & 0.017 \\
\hline 3 & 5101.2 & 5103.105 & 0.037 \\
\hline
\end{tabular}

